\title{
Modelling of imbibition process in an embankment scale model
}

\author{
Giada M. Rotisciani ${ }^{1, a}$, Francesca Casini ${ }^{2}$, Augusto Desideri ${ }^{1}$ and Giulio Sciarra ${ }^{3}$ \\ ${ }^{1}$ Sapienza Università di Roma, Dipartimento di Ingegneria Strutturale e Geotecnica, 00184 Roma, Italia \\ ${ }^{2}$ Università degli Studi di Roma Tor Vergata, Dipartimento di Ingegneria Civile e Ingegneria Informatica, 00173, Roma, Italia \\ ${ }^{3}$ Sapienza Università di Roma, Dipartimento Ingegneria Chimica Materiali Ambiente, 00184 Roma, Italia
}

\begin{abstract}
This paper aims to investigate the hydro-mechanical behaviour of a loosely compacted embankment during an inundation event. This study is based on the results of a centrifuge test carried out on a small-scale embankment model made of an artificially compacted clay-sand mixture. The wetting-induced displacements are analyzed and interpreted by means of a constitutive model adapted to unsaturated conditions. The numerical predictions are presented in terms of time evolutions of settlements, as well as, spatial distributions of vertical displacements. These profiles are compared to those experimentally observed in order to validate the predictive capabilities of the model on a boundary value problem. Moreover, the stress paths followed by elementary soil elements located at different depths are analyzed to emphasize the stress and strain variations due to capillary rise.
\end{abstract}

\section{Introduction}

Soils used in the construction of roadway and railway embankments are usually in partial saturation regime. Once wetted, these materials can collapse causing extensive crest settlements. The triggering of the socalled volumetric collapse is controlled by several factors and, in particular, by the dry density and water content after compaction.

In this study, the centrifuge test conducted by [1] on an embankment scale model is numerically simulated. The back analyses of the experimental results aims to investigate the wetting-induced deformations and the evolution of the stress state due to capillary rise. The material used in the physical modelling is a sand-clay mixture compacted on the dry side of the optimum Proctor curve, at low compaction rate, to emphasize the settlements. The hydro-mechanical behaviour of the solid skeleton is described using the Modified Cam-Clay model extended to unsaturated conditions ([2]). The ability of this model to reproduce the response of elementary soil volumes has been verified over the years by several authors ([3], [4], [5]). The model has been validated also in boundary value problems such as a real dam by [6], and the back analyses of a shallow tunnel ([7]).

The numerical results presented here highlight the satisfactory capabilities of the Modified Cam Clay model in reproducing the effects induced by imbibition processes. The numerical results, validated against the experimental results, represent an important instrument to analyse the stress state evolution during an inundation event.

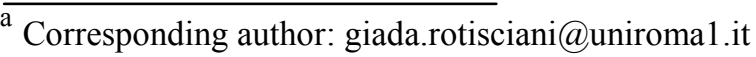

The paper is organized as follows. In the first part, the hydro-mechanical properties of the material, the sequence of the test and the experimental data are summarized. In the second part, the constitutive laws for solid and fluid phases are briefly described together with the main numerical results. In particular, the predicted settlement profiles are compared with the measurements in order to verify the predictive capabilities of the model. Finally, the stress paths resulting from the imbibition process and the volumetric behaviour are analysed with reference to two different soil elements.

\section{Centrifuge test}

The sequence of the test and the experimental data are briefly described in this section. More details on the centrifuge modelling can be found in [1].

The test is conducted at $100 \mathrm{~g}$ on an half embankment model scale of $21 \mathrm{~cm}$ in height made of $40 \%$ Speswhite kaolin and $60 \%$ Fontainebleau sand. The slope is inclined at $33^{\circ}$ to the horizontal plane, according to the classical inclination for real road embankments. The final lengths of the base and the crest are 60 and $28.5 \mathrm{~cm}$, respectively (Fig. 1).

The hydro-mechanical properties of the material, indicated in the following as SCM, are a combination of those of clayey and sandy soils. The standard Proctor curve is similar to that of fine grained soils and provides an optimum water content $w_{\text {opt }}$ of $13.5 \%$ and a maximum dry density $\rho_{M D D}$ of $1.87 \mathrm{~kg} / \mathrm{m}^{3}$. 


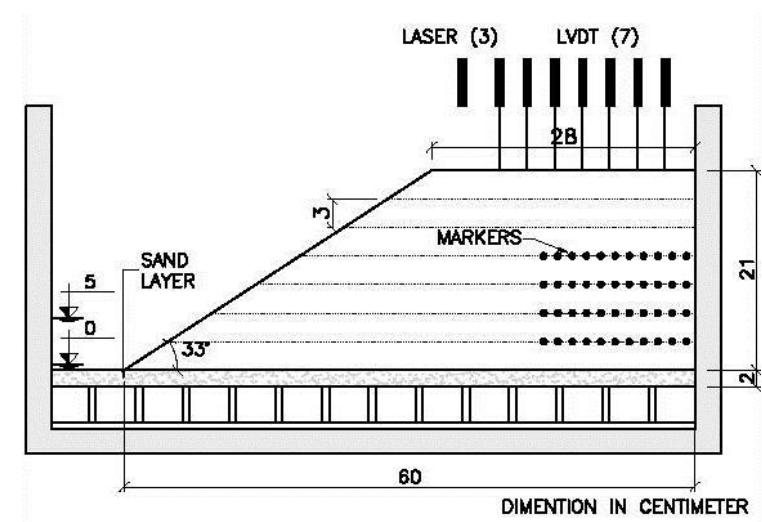

Figure 1. Cross section of the embankment model.

Similarly, the SCM behaviour along stress paths imposed by oedometric conditions does not qualitatively differ to that of clayey soils. The 1-D compression tests are performed by step at the target total vertical stress in saturated conditions and at constant water content for unsaturated samples. The water retention curve (WRC), depicted in Fig. 2 in the plane saturation degree $S_{r}$ versus suction $s$ is obtained using the osmotic method. The WRC exhibited by the material is typical of coarse grained soils, while the compressibility is similar to fine grained soils.

The model is built inside the strong-box in seven successive sub-layers, each approximately $3 \mathrm{~cm}$ thick. The SCM, with a water content of $10.5 \%$, is compacted on the dry side of the Proctor curve to emphasize the displacement phenomena caused by wetting. A sand layer $2 \mathrm{~cm}$ thick is located on the bottom of the strong-box and is separated from the SCM layer by geotextile.

After deposition and compaction, a portion of SCM layer is removed to form a slope inclined at $33^{\circ}$ to the horizontal plane. Upon ending the model preparation, the centrifuge acceleration is increased up to $100 \mathrm{~g}$ (Phase 1). Once stationary conditions are reached, the strong-box is hydraulically connected to a water tank and the water table is constantly maintained just above the sandembankment interface level (Phase 2).

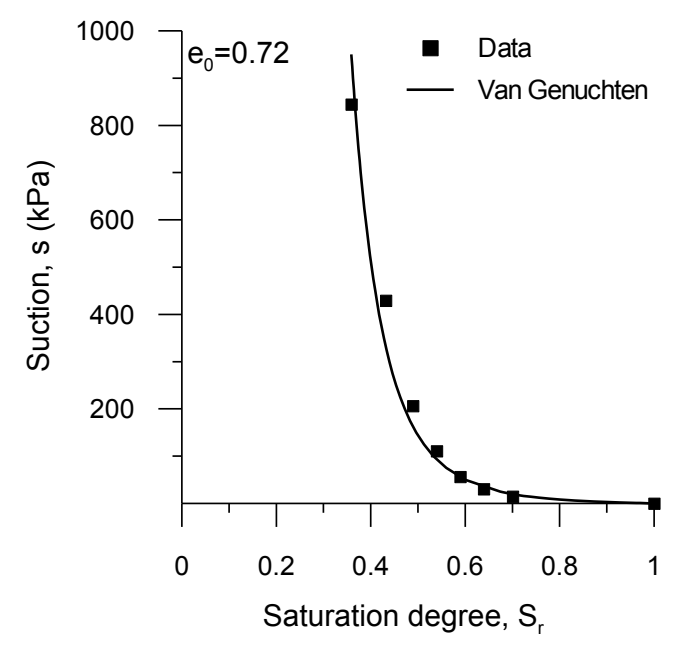

Figure 2. SCM water retention curve.
Finally, the water table inside the main compartment is raised up to $5 \mathrm{~cm}$ above the base (Phase 3 ).

The crest vertical displacements are recorded by three laser transducers, installed close to the crest slope, and seven regularly spaced LVDTs, installed in the central portion of the embankment top. The centrifuge is equipped with a digital video camera to monitor the soil movements during the test. The image analysis allows to determine the vertical displacements of the reference points located inside the embankment body (see Fig. 1).

Fig. 3 illustrates time evolution of the crest vertical displacements due to inundation. The zero values correspond to the settlements registered once ended the in-flight phase. The first imbibition event (Phase 2) induces settlements of about $5 \mathrm{~mm}$, uniformly distributed on the embankment crest. The magnitude of the settlements increase in the Phase 3, when the water table progressively rises up to $5 \mathrm{~mm}$ above the base. The stationary values range from 12 to $14 \mathrm{~mm}$, depending on the considered position. More specifically, the settlements are greater and develop more quickly near the slope than close to the axis of symmetry.

Fig. 5a illustrates the spatial distribution of the wetting-induced settlements, once ended the Phases 2 and 3. The vertical deformations mainly occur in the lower part of the embankment and the magnitude of the settlements appreciably increases as the water table moves upwards to $5 \mathrm{~cm}$ above the bottom. Similarly, volume changes mainly occur in the deep sub-layers and their magnitude increases as the embankment is inundated.

\section{Numerical modelling}

The centrifuge test has been numerically simulated using the commercial code Abaqus/Standard at the model scale. The plane strain simulation reproduces faithfully the geometry and the sequence of the test. In this section, after a brief description of the numerical model, the main numerical results are analyzed.

\subsection{Numerical model}

The soil layer is discretized by a finite element mesh 23 $\mathrm{cm}$ high and $60 \mathrm{~cm}$ wide with element size ranging from $(0.5 \times 0.5)$ to $(1.0 \times 0.5) \mathrm{cm}^{2}$. The adopted mesh is stable and accurate; it is mostly independent by element size. The nodes located along the vertical sides can move vertically while the nodes located along the base are constrained in both directions. Regarding hydraulic boundary conditions, fluid flow is prevented through all external surfaces.

The contact surface between the embankment and the sand base layer is modelled as an elastic-perfectly plastic interface with purely frictional Mohr Coulomb criterion.

The mechanical behaviour of the SCM is described using the Modified Cam-Clay model (MCCM) extended to unsaturated conditions following the approach proposed by [2].

This model is formulated within the framework of critical state soil mechanics in terms of Bishop effective 
stresses: $\sigma_{i j}^{\prime}=\sigma_{i j}-u_{a} \delta_{i j}+\chi\left(u_{a}-u_{w}\right) \delta_{i j}$, where $\sigma_{i j}$ is the total stress, $\chi$ coincides with the saturation degree, $\left(u_{\mathrm{a}}-u_{\mathrm{w}}\right)$ is the matrix suction and $\delta_{i j}$ is the Kronecker delta. MCCM is an elastic-plastic constitutive model, commonly used to reproduce the stress-strain response of fine grained soils. A detailed description of the mathematical formulation and the characteristics of this model can be found in $[3,5]$.

This model differs from that used for saturated soils in the hardening rule. In MCCM, the evolution of the yield surface is controlled by the increments of the plastic volumetric deformation and saturation degree. The introduction of the explicit dependence from $S_{r}$ is needed to reproduce some specific aspects of the hydromechanical behaviour of unsaturated soils, such as the increase in the apparent preconsolidation stress with suction and the collapse upon wetting.

MCCM is completely defined by the six constitutive parameters summarized in Table 1 together with the hydraulic properties. More specifically, the constitutive parameters are: the slope of the unloading-reloading line URL in the compressibility plane (in semi-log scale) $\kappa$, the slope and the position of the saturated normal consolidation line NCL in the same plane, $\lambda$ and $N_{0}$; Poisson's ratio $v$, the slope of the critical state line in the stress invariant plane $M$ and, finally, the parameter $b$ that controls the relative position of the NCL associated to the current value of $S_{r}$ with respect to the saturated NCL.

The model is calibrated against the results of oedometric (OED) and cone penetration tests (CPT). The first ones are conducted on saturated and unsaturated soil samples; the CPT are carried out on an embankment scale model similar to those used in the centrifuge test.

The fluid flow obeys the well-known Richards equations:

$$
\nabla\left[\frac{k\left(S_{r}\right)}{\gamma_{w}} \nabla\left(s+\gamma_{w} z\right)\right]=\frac{\partial \theta}{\partial t}
$$

where $k\left(S_{r}\right)$ represents the evolution law of the permeability with the saturation degree, $\gamma_{w}$ is the specific weight of water and $\theta$ is the volumetric water content, defined as ratio between volume of water and total volume.

Table 1. SCM mechanical and hydraulic properties.

\begin{tabular}{|c|c|c|c|}
\hline \multicolumn{2}{|c|}{$\begin{array}{c}\text { Mechanical } \\
\text { parameters }\end{array}$} & \multicolumn{2}{c|}{$\begin{array}{c}\text { Hydraulic } \\
\text { parameters }\end{array}$} \\
\cline { 1 - 2 }$\lambda$ & 0.085 & $\alpha$ & 0.35 \\
\hline$\kappa$ & 0.025 & $n$ & 1.17 \\
\hline$N_{0}$ & 1.94 & $m$ & 0.15 \\
\cline { 1 - 2 }$v$ & 0.25 & \multirow{2}{*}{$k_{\text {sat }}(\mathrm{m} / \mathrm{s})$} & $510^{-8}$ \\
\cline { 1 - 2 }$M$ & 1.11 & \multicolumn{2}{|c}{} \\
\cline { 1 - 2 }$b$ & 7.4 & \multicolumn{2}{|c}{} \\
\cline { 1 - 2 } & \multicolumn{2}{|c}{}
\end{tabular}

$k\left(S_{r}\right)$ is expressed as a power function of the degree of saturation: $k\left(S_{r}\right)=k_{s a t} S_{r}{ }^{\delta}$. The values for $\delta$ and the saturated permeability $k_{\text {sat }}$ are deduced by back-analysing the time evolution of the crest vertical displacements (see Fig.4).

The WRC is fitted with the Van Genucthen expression; the parameters $\alpha, m$ and $n$ are calibrated against the experimental data (see Fig. 3)

$$
S_{r}=\frac{1}{\left[1+(\alpha s)^{n}\right]^{m}}
$$

The numerical analysis accurately reproduces the sequence of the test and consists of four steps.

Under geostatic conditions, the total vertical stresses are completely negligible as the small dimensions of the embankment. The pore water pressures vary linearly with depth and assume an average value of $675 \mathrm{kPa}$, corresponding to a water content of $10.5 \%$. The effective horizontal stresses are expressed as a function of the effective vertical stresses through the earth pressure coefficient $K_{0}$, placed equal to the unity.

To form the embankment, the mesh elements located above a surface inclined at $33^{\circ}$ to horizontal plane are removed (Phase 0 ). The $\boldsymbol{g}$ level is increased by a factor of 100 , coherently to the centrifuge test (Phase 1). Then, null pore water pressure is prescribed at the embankment base to simulate the rising of the water table (Phase 2). Finally, the inundation event is modelled by imposing a constant pore water pressure of $50 \mathrm{kPa}$ at the sandembankment interface level and applying a hydrostatic pore water pressure distribution on the slope, according to a water table located $5 \mathrm{~cm}$ above the base (Phase 3 ).

\subsection{Numerical results}

Time evolution of the predicted crest settlements is shown in Fig. 3 together with the data registered by the transducers. The numerical results refer to two nodes located at the top of the soil domain along the slope and the axis of symmetry. Globally, the model responses fit well the experimental curves.

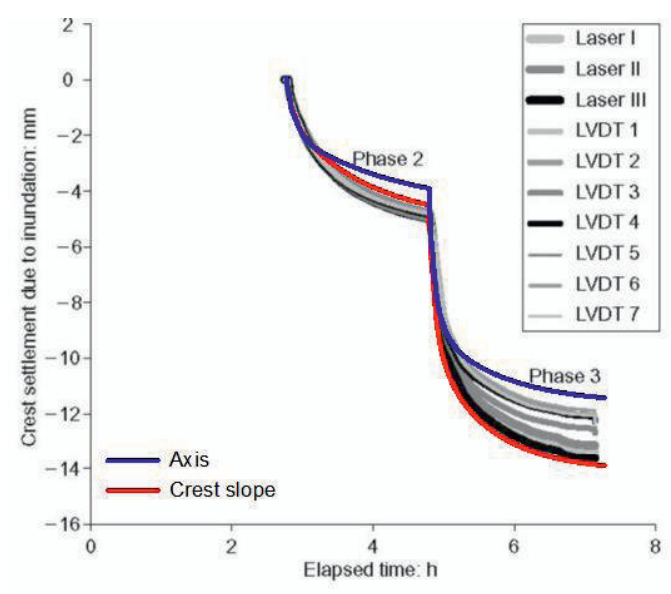

Figure 3. Time evolution of crest settlements. 
In the Phase 2, the vertical displacements increase in magnitude up to values ranging from 4 to $5 \mathrm{~mm}$. Differently to what is observed in the test, there is a small difference between the predicted settlements at the crest slope and the axis. In the Phase 3, the vertical displacements increase again in magnitude attaining values in the range of $11-14 \mathrm{~mm}$. Similarly to the measurements, the predicted vertical displacements are more extensive and develop more quickly at the crest slope than at the axis. In this phase, the wetting-induced settlements are accompanied by significant lateral displacements, whose magnitude increases approaching the slope. As a consequence, the crest settlements near the axis, where no horizontal displacement is allowed, are smaller than those predicted at the slope, where lateral and vertical movements are combined.

The predicted settlements profiles are depicted in Fig. 4 together with the measurements. The numerical results refer to a vertical axis $0.5 \mathrm{~cm}$ far from the axis of symmetry. The satisfactory match between model predictions and experimental data confirms the ability of the Modified Cam-Clay model in reproducing wettinginduced deformations also in a boundary value problem.

The magnitude of the predicted vertical displacements appreciably increases as the water table rises up to $5 \mathrm{~cm}$ above the base (see Fig. 5). Despite the quantitative differences, the $w$ profiles exhibit similar trends in the Phases 2 and 3. The settlements increase in the lower part of the embankment and keep an almost constant value in the upper part. Comparing the model predictions with the data, the major differences can be found in the upper part. Here, the MCCM predicts a soil response appreciably stiffer than that experimentally observed.

Upon validating the constitutive model, the hydromechanical response of the collapsible soil is analyzed in detail. The changes in the pore-water pressure $u_{\mathrm{w}}$ and degree of saturation, as well as, the stress paths and the deformations induced by capillary rise are examined with reference to two elements located at different depths along a vertical axis $5 \mathrm{~cm}$ far from the axis of symmetry $(z=2.75$ and $14.75 \mathrm{~cm})$.

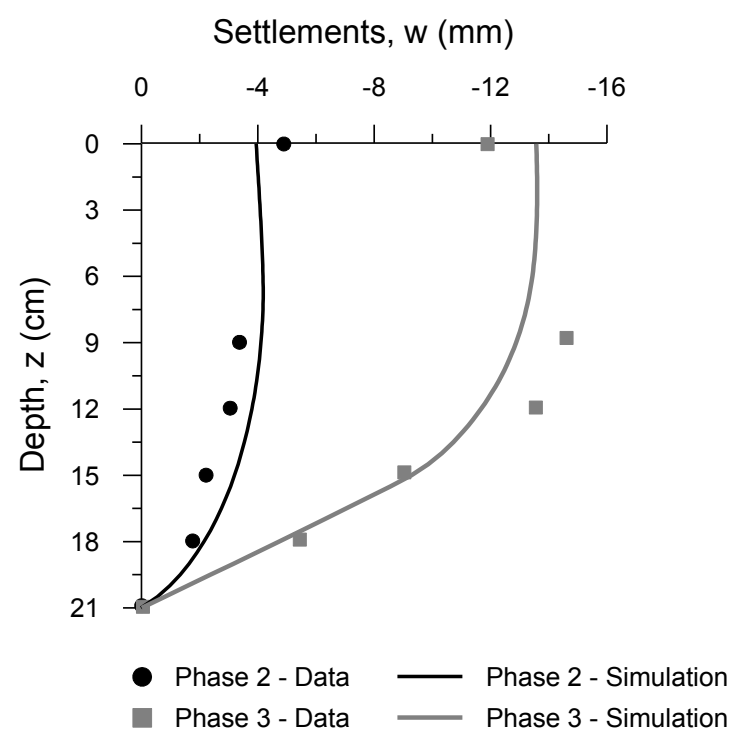

As shown in Fig. 5, the application of the $g$-level entails a rotation of the $u_{\mathrm{w}}$ profile in a counter clockwise direction. This implies a reduction of the pore-water pressure of about $36 \mathrm{kPa}$ in the shallow element and an increment of $84 \mathrm{kPa}$ in the deep one. Despite the variations of $u_{\mathrm{w}}$, there is no appreciable change in the $S_{r}$ profile. This is mainly due to the shape of WRC and the initial conditions, in terms of saturation degree and suction level. In the in-flight phase, the point representative for the current conditions in both considered elements moves along the vertical branch of the WRC, where large variations of suction level are associated to small changes in $S_{r}$.

In the Phase 2, the pore-water pressures progressively increase and, once reached stationary conditions, exhibit a hydrostatic profile with a water table located at the bottom of the soil domain. Regardless of the considered element, $u_{\mathrm{w}}$ increases of about $520 \mathrm{kPa}$. Conversely, the variation in $S_{r}$ depends on depth and is more pronounced in the deep element than in the shallow one. This result can be attributed to the shape of WRC and the differences in the suction level at the beginning of the Phase 2 . Despite the increase of the pore-water pressure associated to the rising of the water level, the point representative for the shallow element lies again on the vertical branch of WRC. Conversely, the suction level in the deep element exceeds the maximum curvature point of the WRC and reaches the horizontal branch of the curve, where small variations of suction entail large changes in the degree of saturation.

In the Phase 3 , as the water infiltrates through the base and the slope, the water table progressively rises within the embankment body and the pore-water pressures increase up to their hydrostatic stationary values. Consequently, the saturation degree increases and complete saturation is reached in soil elements located below the current position of the water table.

The above mentioned differences in the evolution of $S_{r}$ significantly affect the hydro-mechanical responses of the two considered elements. The changes in the stress state and the corresponding volumetric deformations of the deep element are shown in Fig. 6 in terms of mean effective stress $p^{\prime}$, deviator $q$ and specific volume $v$.

The initial stress state lies on the $p^{\prime}$-axis inside the current yield surface (point A), as expected in highly over-consolidated soils $\left(K_{0}=1\right)$.

The application of the centrifuge acceleration induces an increase of the gravity loading exerted on the solid skeleton and no appreciable change in the degree of saturation. Because of the oedometric boundary conditions, the stress state is forced to move along a stress path, whose slope is prescribed by the elastic coefficient $K_{0}^{e l}=v /(1-v)$. Once reached the current yield surface, the stress path changes in slope and progressively tends towards the $K_{0}$ line (branch AB). The volumetric behaviour is initially controlled by the slope of the URL and, then, by NCL in oedometric conditions associated to the current saturation degree.

Figure 4. Spatial distribution of settlements. 

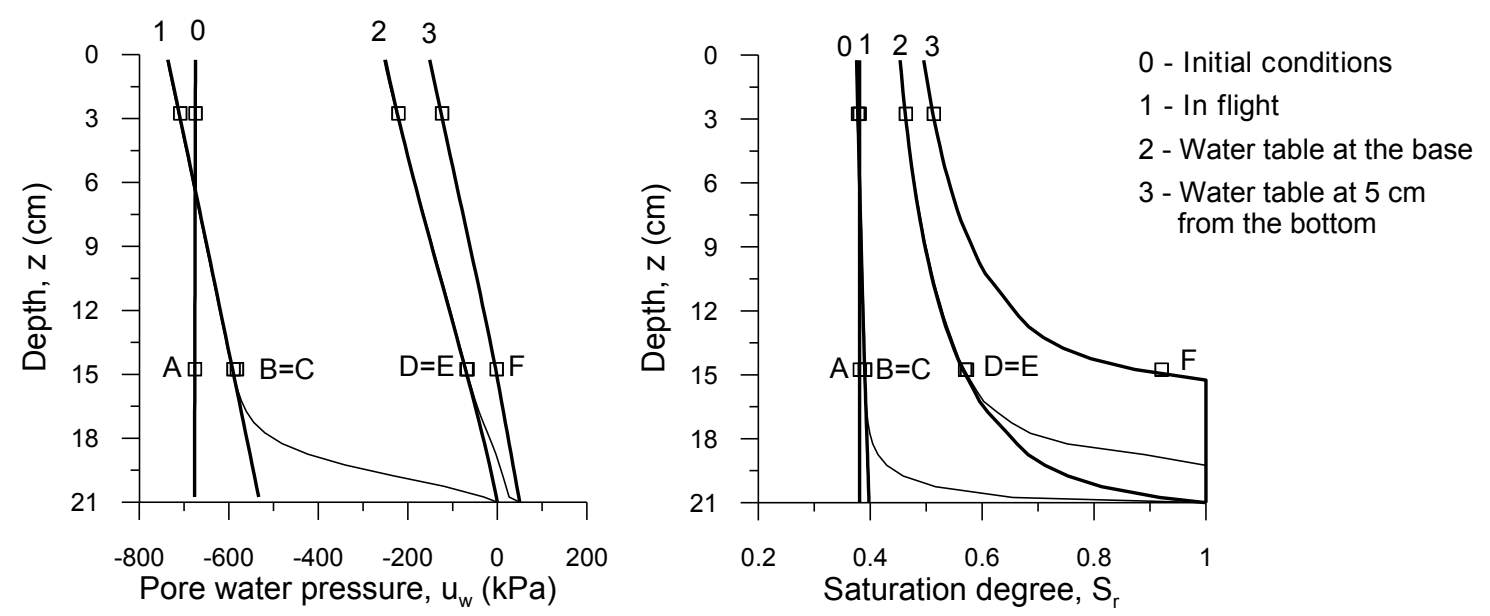

Figure 5. Time evolution of the pore water pressure and saturation degree profiles.

When null pore-water pressure is prescribed at the base, the mean effective stress decreases and the deviator slightly increases (branch BC). It is to notice that this increment of the stress ratio $\eta=q / p^{\prime}$ occurs when the capillary front is still below the considered element (see Fig. 5). The propagation of the front is accompanied by a variation of the horizontal stress distribution due to the volumetric collapse of the deep elements. This variation involves also the soil region just above the current position of the front and justifies the reduction of the horizontal stress under a constant vertical load in the considered element. Once the front reaches the deep element, the stress ratio starts to decrease. Despite the stress release, the skinkage of the yield surface due to the increase of $S_{r}$ keeps the stress state constantly in elasticplastic regime (branch $\mathrm{CD}$ ). As a consequence, the stress path tends towards the $K_{0}$-line and solid skeleton collapses. Significant volume changes occur only when $S_{r}$ exceeds 0.5 , which corresponds to the maximum curvature point of WRC. For $S_{r}$ lower that this value, the positive volumetric deformations are well-balanced by the elastic swelling and the soil element deforms under a quasi constant volume. During the inundation event, the soil response is definitely similar to that observed in the Phase 2. As soon as the water starts to infiltrate in the embankment body through the bottom and the slope, the horizontal stress in the considered element decreases under a constant vertical stress. This implies an increment of the stress ratio and, consequently, a change in volume of the current yield surface (branch DE). When the front reaches the element, both stress invariants decrease, the elastic domain skinks and the volumetric collapse occurs (branch EF).

Consider now the responses of the shallow element.

At $1 \boldsymbol{g}$, the mean effective stress is given by the suction level scaled by the saturation degree and, hence, there is no appreciable difference in the initial stress state between the deep and shallow elements. The application of the centrifuge acceleration induces an increment of the gravity loading exerted on the solid skeleton that is more extensive in the deep than in the shallow element. As a consequence, the evolution of the stress state in the deep element provokes the occurrence of plastic deformations while the shallow element behaves as an elastic material.

In the Phase 2, the stress state of the deep element constantly lies on the current yield surface. Conversely, the stress state of the shallow element moves again inside the elastic domain and its evolution is accompanied by positive variations in volume.
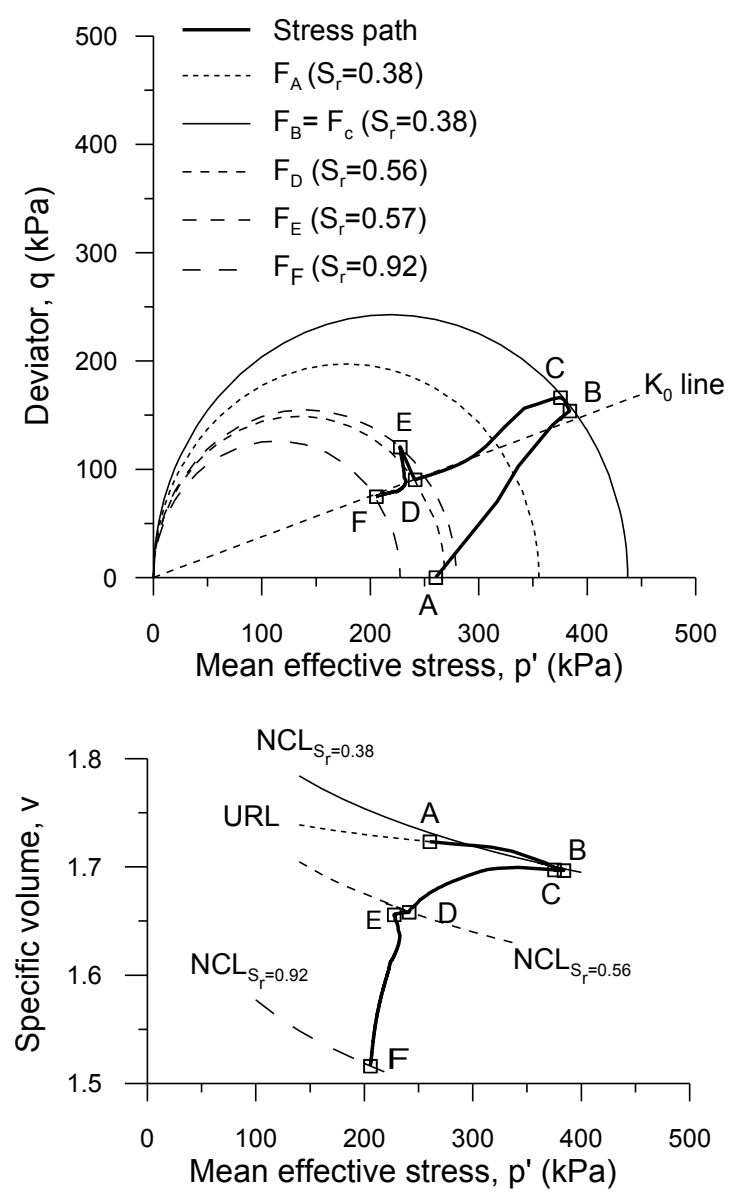

Figure 6. Stress paths and volumetric behaviour of deep elements $(z=14.75 \mathrm{~cm})$. 
In the Phase 3 , the ellipse captures the current stress state and irreversible deformations start to develop. However, differently to the deep element, the shallow element swells again because the elastic strains are greater than that associated to the volumetric collapse.

Once calibrated and validated, the numerical model has been adopted to investigate the influence of the base friction angle on the embankment response. A number of four additional numerical analyses has been performed modifying the contact friction angle between the sand layer and embankment $\varphi_{\mathrm{c}}$ in the range of $10-35^{\circ}$.

The results are depicted in Fig. 7, in terms of spatial distribution of the crest vertical and horizontal, $u$, displacements once reached the final stationary conditions. The comparison shows that there is no appreciable change in the embankment response when $\varphi_{\mathrm{c}}$ assumes values greater than $20^{\circ}$. Conversely, when $\varphi_{\mathrm{c}}$ is lower than $20^{\circ}$, the magnitude of the vertical and horizontal displacements depends on the base friction angle and, in particular, increases as $\varphi_{\mathrm{c}}$ decreases. However, it is to notice that significant variations of $u$ and $w$ occur only when $\varphi_{\mathrm{c}}$ becomes lower than $15^{\circ}$. These parametric analyses confirm the influence of the roughness of the contact surface on the performance of the embankment which it is non linear with the contact friction angle.

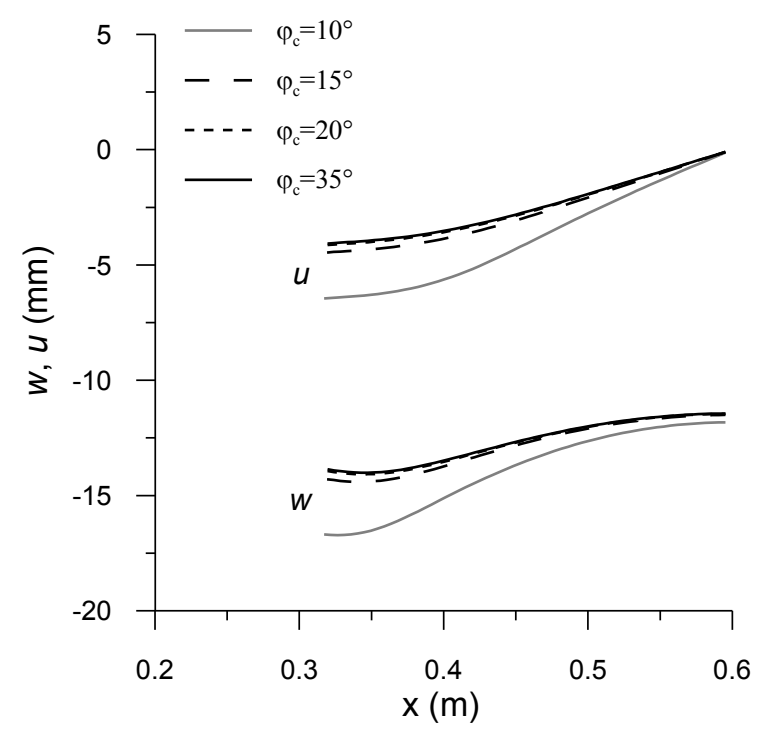

Figure 7. Spatial distribution of the crest vertical and horizontal displacements.

\section{Conclusion}

The results presented in this paper confirm the ability of the Modified Cam-Clay model to accurately predict wetting-induced deformations. The model responses, in terms of time evolution of crest settlements and spatial distribution of settlements and void ratio, well match the experimental data. Moreover, the numerical results underline the influence of the initial conditions, in terms of suction level and saturation degree, and the shape of the WRC on the occurrence of the volumetric collapse. The increase of $S_{r}$ causes appreciable volumetric deformations only in the lower part of the embankment, where the suction level exceeds the maximum curvature point of the WRC. Therefore, to limit the risks associated to the triggering of the volumetric collapse, particular attention should be paid on the compaction procedure of the deep sub-layers. Here, the dry density should be maximized by keeping the water content close to the optimum value and/or increasing the compaction energy.

\section{References}

1. L. Thorel, V. Ferber, B. Caicedo, I.M. Khokhar, Géotechnique 61, 5, 404-420 (2011).

2. C. Jommi, Experimental Evidence and Theoretical Approaches in Unsaturated Soils (A. Tarantino and C. Mancuso ed.), 139-153 (2000).

3. R. Tamagnini, Géotechnique 54, 3, 223-228 (2004).

4. F. Casini, Can. Geot. J. 49, 8, 954-960 (2012).

5. G. M. Rotisciani, G. Sciarra, F. Casini, A. Desideri, Int. J. Numer. Anal. Meth. Geomech., 39, 1212-1234 (2014).

6. A. Burghignoli, A. Desideri, and R. Tamagnini, $3^{\text {th }}$ Int. Conf. Unsat. Soils 1, 221-225 (2002).

7. E. Soranzo, R. Tamagnini, W. Wu, Géotechnique 65, 6, 454-467 (2015). 